\title{
PROGRAMA PILOTO DE VIVIENDA SOSTENIBLE
}

Arq. Fabio Claros Lamus

Arq.Dione Esmeralda Pérez

Briceño*
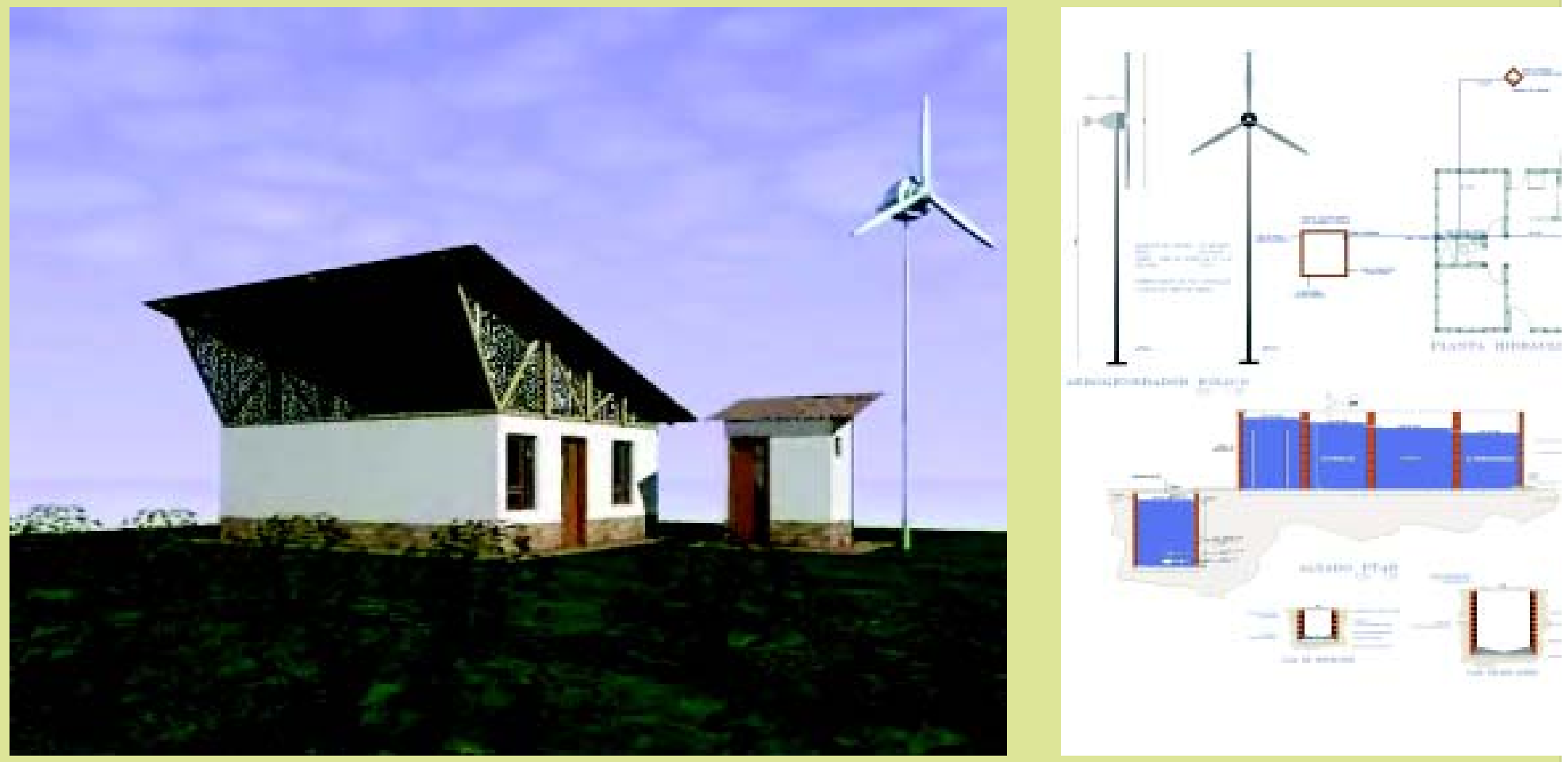

\section{Resumen}

El proyecto quiere iniciar un proceso de cambio en la conceptualización y construcción de áreas residenciales de interés social, mediante la ejecución de un proyecto piloto, para conseguir que sean ambientalmente sostenibles y mejorar la calidad de vida de sus propietarios. Se parte de un modelo en un sistema constructivo, modular, económico y de fácil implantación; que abarca tres aspectos esenciales: la ecológica (utilizar la productividad de la naturaleza sin dañarla), la económica (aseguramiento de los recursos necesarios para el consumo humano), y la social (fomentar el beneficio social a través del confort).

\section{Palabras Clave}

Desarrollo sostenible, vivienda, aplicación de tecnologías limpias, cultivos orgánicos, organización de la comunidad en cooperativa 

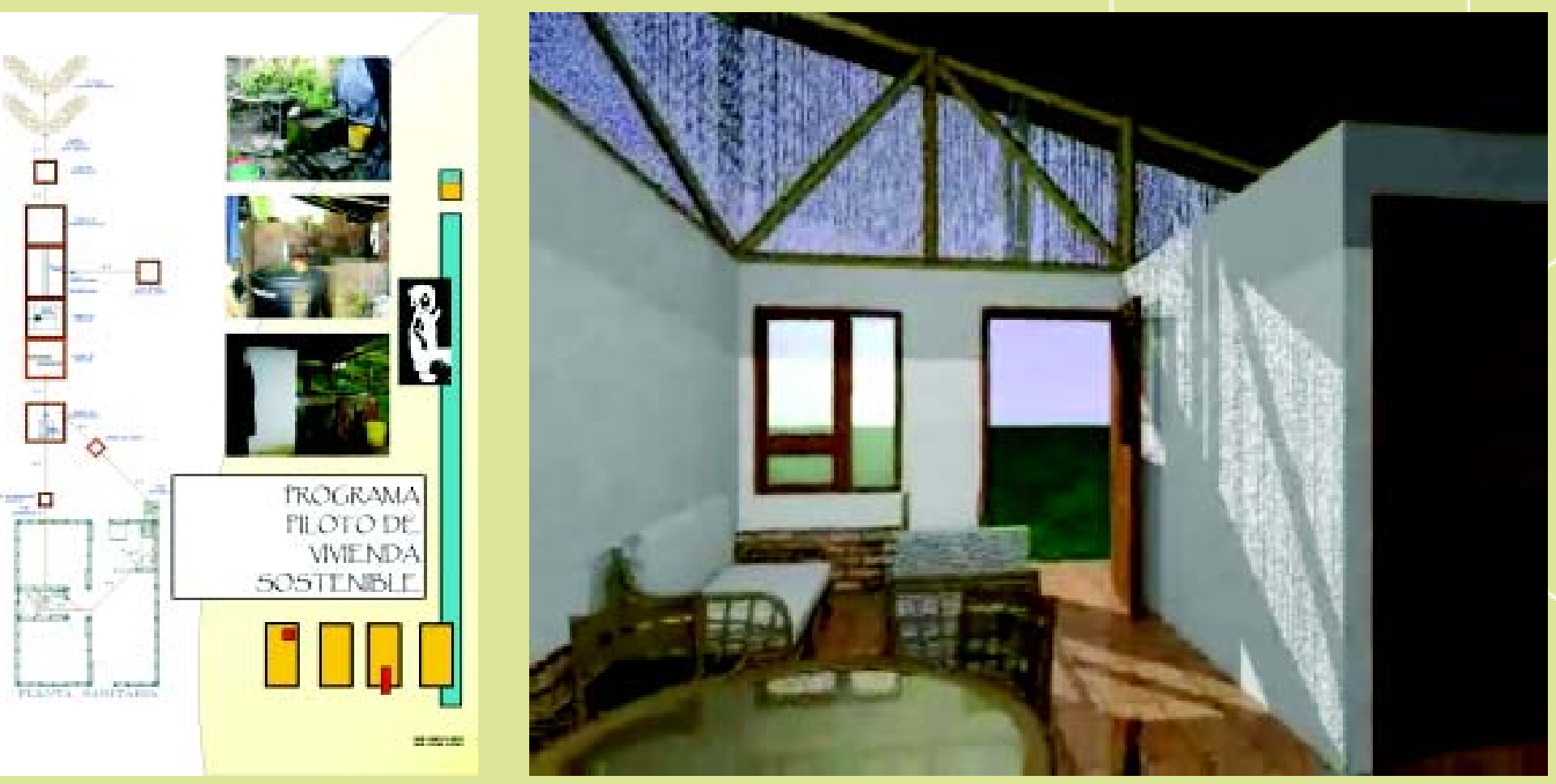

\section{Abstract}

The project wants to initiate a process of change in the conceptualization and construction of social interest's residential areas, by means of the execution of a pilot project, to get them to be environmentally sustainable and to improve the quality of life of its proprietors. It starts from a model in a constructive system, modular, economic and easy to implant, that includes three essential aspects: the ecological one (to use the productivity of the nature without damaging it), the economic one (securing of the necessary resources for the human consumption), and the social one (to foment the social benefit through comfort).

\section{Key Words}

Sustainable development, housing, application of clean technologies, organic cultures, cooperative organization of the community 


\section{Información general del proyecto}

Programa piloto de vivienda sostenible rural o suburbana, mediante el aprovechamiento de energía eólica y un sistema de tratamiento de aguas residuales.

FABIO CLAROS LAMUS, Universidad Santo Tomás

PONENTE, INVESTIGADOR

DIONE ESMERALDA PÉREZ BRICEÑO

ARQUITECTA, Universidad Santo Tomás, INVESTIGADOR

Iniciación del proyecto: 02/01/1999

Terminación del proyecto: 30/08/2005

\section{Propuesta}

La necesidad de establecer acciones y metas que permitan un aprovechamiento racional de los recursos, enmarcados en un "DESARROLLO" que preserve el medio físico y biológico, llamado así mismo "CONSERVACION" situaciones que nos permitirán llegar al desarrollo sostenible, establecer la posibilidad real de utilizar los recursos de forma permanente, preservar e interactuar con el medio circundante.

Bajo esta premisa inicia el proyecto de investigación, un programa piloto de vivienda sostenible que integra el manejo de residuos sólidos, líquidos y la energía eólica. Se pretende acortar el distanciamiento del Estado al ciudadano, al cual le añaden "soluciones" a problemas de salud, agua potable y/o vivienda. Para ello el proyecto busca establecer un ciclo de estas necesidades inherentes a la humanidad. Es decir, un ecosistema, que inicia con la producción de una vivienda digna, que actúa como núcleo, con tecnologías alternativas y limpias, donde el control de vectores (enfermedades), y el derecho a vivir dignamente se hacen evidentes, para suplir una necesidad común como lo es el empleo.

Para ello se propone una vivienda en bahareque embutido o encementado, con una organización espacial y de confort, que funciona con un aerogenerador eólico (molino de viento), el cual abastece de energía eléctrica a la vivienda, pero: ¿Cómo suplir la falta de empleo? La respuesta los cultivos orgánicos (stevia o cacao), que con ayuda de entidades como FINAGRO, establecen seguimientos y posterior aval, para su producción masiva, y para su exportación, tal fin se logra mediante la organización de la comunidad en una cooperativa de trabajo asociado, que permita comercializar sus productos. En la integración evidente de varios programas del Estado, radica el éxito del presente proyecto de investigación, donde la vivienda ecosistémica reproduce y soluciona los requerimientos objeto del proyecto.

El objetivo general es desarrollar una vivienda rural o suburbana, de fácil adaptación que permita integrar el aprovechamiento del suelo y la adquisición de servicios básicos de forma sostenible, mediante la aplicación de tecnologías limpias. Con unos objetivos específicos como: crear las condiciones que propicien la siembra de cultivos orgánicos, bajo la premisa de cero desperdicio y conservar el ecosistema. Crear un sentido de apropiación, a nivel social, económico y ambiental, con el fin último de establecer mejores condiciones de vida. Producir una vivienda rural o suburbana, que funcione con energía eólica, y un manejo racional sostenible de residuos sólidos y líquidos mediante pequeñas plantas residenciales de tratamiento.

El proyecto quiere iniciar un proceso de cambio en la conceptualización y construcción de áreas residenciales de interés social, mediante la ejecución de un proyecto piloto, para conseguir que sean ambientalmente sostenibles y mejorar la calidad de vida de sus propietarios. Se parte de un modelo en un sistema constructivo, modular, económico y de fácil implantación; que abarca tres aspectos esenciales: la ecológica (utilizar la productividad de 
la naturaleza sin dañarla), la económica (aseguramiento de los recursos necesarios para el consumo humano), y la social (fomentar el beneficio social a través del confort).

Para ello se acompaña de una capacitación en agricultura orgánica, enfocados hacia la stevia o cacao orgánico. Su fin último es la no utilización de insumos externos, a cambio de realizar las prácticas fitosanitarias y de producción a partir de procesos y controles naturales o biológicos, en busca de obtener producciones limpias, de mayor calidad nutricional, que no generen problemas a la salud humana, y preserven el ecosistema.

Estas aplicaciones conllevan una serie de resultados: Reducción en un 30\% del concreto usualmente empleado en este tipo de obras. Disminución esperada en cerca de un 20\% en el consumo de agua potable por casa. Reducción, al menos, de un 15\% en el costo de las viviendas. Afectación mínima al medio ambiente, y manejo integral de los desechos sólidos y líquidos. Dejar una comunidad organizada y consciente, dispuesta a asumir sus propias responsabilidades, como base para la superación permanente. Reducción económica de los servicios básicos. Participación de la comunidad, en la ejecución de su proyecto habitacional. Aplicación de conceptos de integración de comunidad, hábitat ambiental y desarrollo socioeconómico local. Búsqueda de una economía local sostenible, para lograr un hábitat protegido generador de empleo y riqueza, para un desarrollo rural. Realizar jornadas de educación ambiental, y jornadas de sensibilización para la población beneficiada.
"Sólo si nos exponemos a la posibilidad de una visión contraria, tendremos la oportunidad de trascender la estrechez de nuestras propias prevenciones"

(H.G. Gadamer 1997)

\section{INTRODUCCIÓN}

La academia despierta el interés y la afinidad del hombre por el hombre de su relación con el contexto para superase o simplemente para caer en un estado de pasividad, una muerte que no entiende de razones de vida o de derechos flagelados, que pregona renunciar a una vida digna. He aquí una nueva forma de analizar los proyectos de vivienda y salud, donde estamos acostumbrados a emitir productos industrializados enfocados a contaminar el medio ambiente con materiales y sistemas constructivos, con soluciones aparentes de problemas sociales, políticos y ambientales. Donde el sueño de un hábitat integral converge en la aplicación del DESARROLLO SOSTENIBLE, y permite iniciar un proceso de investigación donde las experiencias de otros y el ensayo de prueba error permitieron dirigir un proyecto en particular.

Bajo esta premisa inicia el programa piloto de vivienda sostenible. Un programa que integra el manejo de residuos sólidos, líquidos y de energía eólica. Cuya intención es acortar el distanciamiento del Estado al ciudadano, al cual le añade "soluciones" a problemas de salud, agua potable y vivienda. Para ello, el proyecto busca establecer un ciclo de estas necesidades inherentes a la humanidad. Es decir, un ecosistema, que se inicia al producir una vivienda digna, que actúa como núcleo, con tecnologías alternativas y limpias, donde el control de vectores (enfermedades), y el derecho a vivir dignamente se hacen evidentes, para suplir una necesidad común como lo es el empleo.

Para ello se propone una vivienda en bahareque embutido o encementado, con una organización espacial y de confort, que funciona con un aerogenador eólico (molino de viento), el cual abastece de energía eléctrica a la vivienda, pero: ¿Cómo suplir la falta de empleo? La respuesta, los cultivos orgánicos (stevia o cacao), que con ayuda de entidades como FINAGRO, establecen seguimientos y posterior aval, para su producción masiva, y exportación, tal fin se logra mediante la organización de la comunidad en una cooperativa de trabajo asociado, que permita comercializar sus productos. Es así como en la integración evidente de varios programas del Estado radica el éxito del presente proyecto de investigación, donde la vivienda ecosistémica reproduce y soluciona los requerimientos objeto del proyecto. 


\section{INDICADORES}

\subsection{MEDIDAS QUE DEBEN SER TOMADAS}

- La degradación de los recursos naturales por el impacto humano, podría controlarse, si se tomaran acciones paralelas que conjuntamente, con un desarrollo económico más justo, informaran a los ciudadanos sobre el impacto que adecuadas políticas para el desarrollo sostenible podrían tener en un futuro casi inmediato. Se plantea una estrategia enfocada en educar, informar, concienciar y contar con la participación social. Éstos son los componentes sobre los que se articula EL PROGRAMA PILOTO DE VIVIENDA SOSTENIBLE.

- No podemos seguir con estrategias de sobre vivencia, debemos establecer acciones y metas que permitan un aprovechamiento racional de los recursos, enmarcados en un "DESARROLLO" que preserve el medio físico y biológico, Ilamado asimismo "CONSERVACIÓN" situación que nos permitirá llegar al desarrollo sostenible, establecer una posibilidad real de utilizar los recursos de forma permanente, para preservar e interactuar con el medio circundante.

\subsection{DESARROLLO SOSTENIBLE}

- De acuerdo a la definición de la Comisión Mundial del Medio Ambiente y del Desarrollo de las Naciones Unidas: "El Desarrollo Sostenible debe satisfacer las necesidades de las generaciones futuras sobre la base de satisfacer sus necesidades presentes. Esto encierra en sí dos conceptos fundamentales: el de "necesidades", en particular, las necesidades esenciales de los pobres, a las que se debería otorgar prioridad preponderante, y la idea de limitaciones impuestas por la tecnología y la organización social entre la capacidad del medio ambiente para satisfacer las necesidades presentes o futuras"

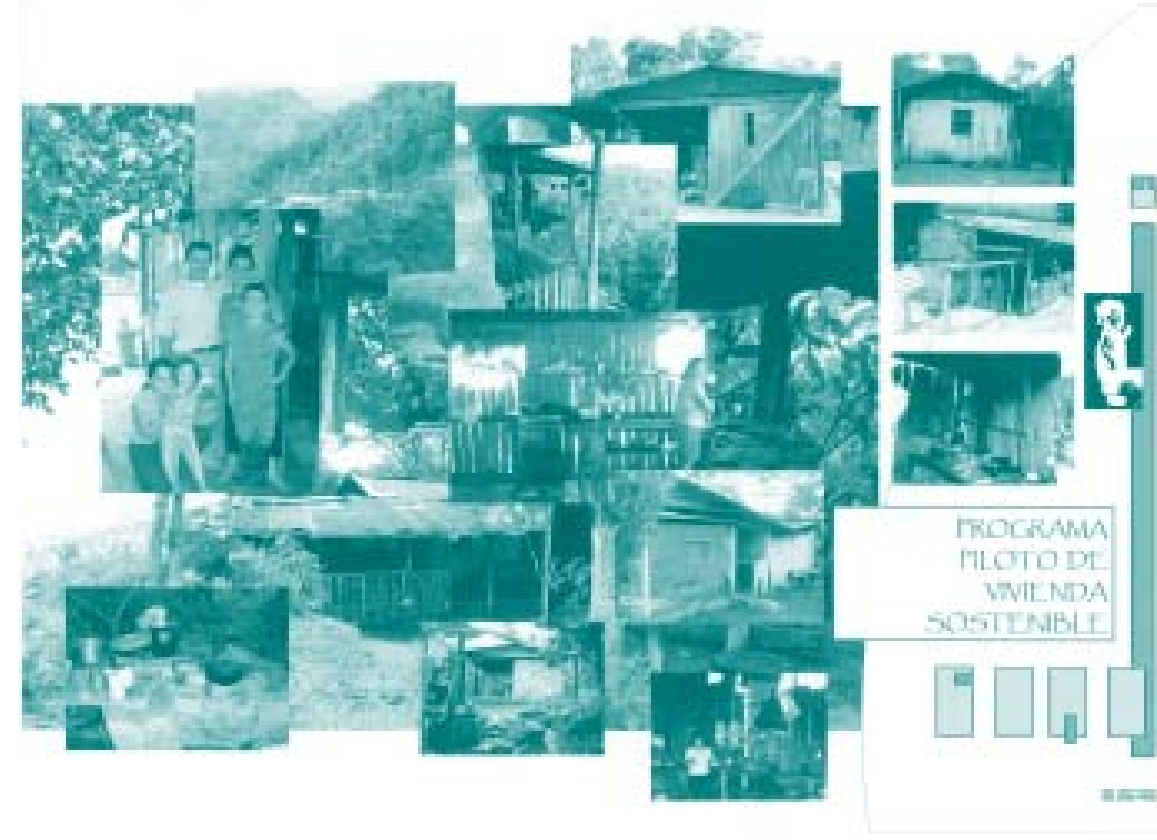




\subsection{IMPLICACIONES}

- Aplicación de conceptos universales de sostenibilidad adaptada a los diseños de los asentamientos humanos, con énfasis en los de interés social.

- Capacitación, organización y participación activa de la comunidad en la toma de decisiones relativas al asentamiento, como medio que propicie su superación permanente en el futuro.

- Reducción de costos y minimización en la afectación al medio, gracias al uso racional de los insumos que se incorporan en la construcción de los asentamientos: recursos materiales, económicos y humanos.

- Industrialización y modulación en el sitio mediante la fábrica autogestionaria de elementos constructivos, con empleo de tecnologías apropiadas basadas en equipos económicos y de fácil manejo, que bajen costos, reduzcan desperdicios y eliminen intermediarios.

- Reducción en el consumo energético mediante el aprovechamiento de energías pasivas.

- Aplicación de sistemas universales de tratamiento de aguas servidas, emplear las fuerzas de la gravedad y los procesos biológicos naturales y económicos de purificación.

- Desarrollo de plantas de tratamiento de aguas negras, que pueden llevarse incluso a nivel unifamiliar, constituidas por sedimentadores primarios, filtros biológicos anaerobios de flujo ascendente y lechos de secado de lodos.

- Consideración del entorno en los diseños: topografía, suelo, condiciones climáticas, vegetación, aspectos culturales y sociales, y otros.

- Propiciar, por medio del diseño y el proceso, el desarrollo de comportamientos sociales deseables: solidaridad, cooperación, creatividad, sentido de comunidad y organización, control social, autoestima.

- Generación de resultados concretos y medibles como base para la definición y cambio de políticas, estrategias y normativas nacionales en procura del desarrollo sostenible de los asentamientos humanos.

- Disposición de parámetros e instrumentos que permitan valorar la sostenibilidad de los asentamientos humanos, como medio de orientación de nuevos diseños y renovación de los existentes.

- Ofrecer a los Gobiernos respuestas tecnológicas y organizativas concretas para la aplicación de los principios de sostenibilidad en el desarrollo de los asentamientos humanos.

\section{BENEFICIOS ALCANZADOS}

- Reducción en un $40 \%$ del costo promedio nacional de urbanización por lote (obras de infraestructura).

- Disminución de las áreas pavimentadas en un 35\% con respecto al promedio nacional. Incremento proporcional en las áreas verdes y parques (arborización urbana).
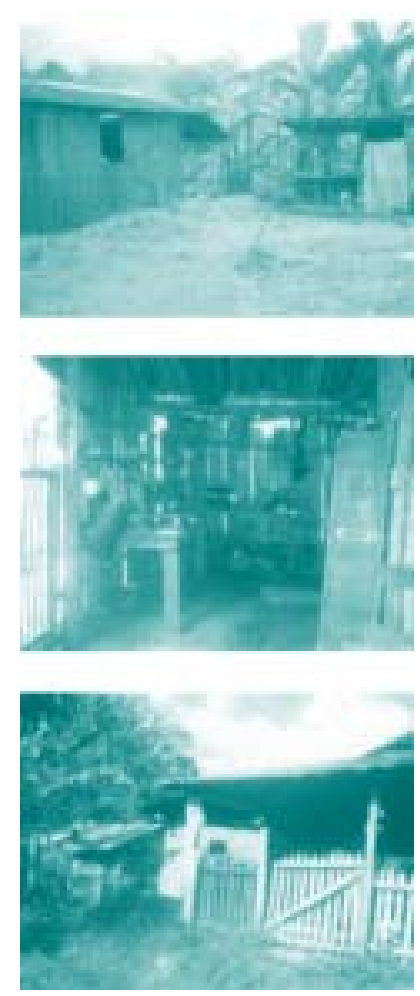
- Reducción en un 30\% del concreto usualmente empleado en este tipo de obras.

- Reducción mayor del 50\% en el costo de las cubiertas de techo.

- Movimientos de tierra llevados al mínimo, respetando el entorno natural.

- Disminución esperada en cerca de un 20\% en el consumo de agua potable por casa, comparado con el promedio nacional.

- Reducción al menos de un 15\% en el costo de las viviendas.

- Afectación mínima al medio ambiente, manejo integral de los desechos sólidos y líquidos.

- Disminución de costos de intermediarios, de transporte, y otros, buscar, mediante procesos sencillos de industrialización y modulación en el sitio con participación autogestionaria, que las comunidades incurran sólo en los gastos indispensables (compra de materia prima directa).

- Desarrollar una comunidad organizada y consciente, dispuesta a asumir sus propias responsabilidades, como base para la superación permanente.

\section{OBJ ETIVO GENERAL}

Desarrollar un programa piloto de vivienda sostenible, que actúe como núcleo integrador de aspectos sociales, económicos y de confort al campesino, mediante un proyecto de fácil adaptación que permita integrar el aprovechamiento del suelo y la adquisición de servicios básicos de forma sostenible, mediante la aplicación de tecnologías limpias.

\subsection{OBJ ETIVOS ESPECÍFICOS}

- Crear las condiciones que propicien la siembra de cultivos orgánicos, bajo la premisa del cero desperdicio y conservar así el ecosistema rural.

- Crear un sentido de pertinencia, a nivel social, económico y ambiental, con el fin último de establecer mejores condiciones de vida.

- Producir una vivienda rural, que funcione con energía eólica, y un manejo racional sostenible de residuos sólidos y líquidos.

\section{VIVIENDA}

- El objetivo general es iniciar un proceso de cambio en la conceptualización y construcción de áreas residenciales de interés social, mediante la ejecución de un proyecto piloto, para conseguir que sean ambientalmente sostenibles y mejorar la calidad de vida de los habitantes.

- Partiendo de un modelo, en un sistema constructivo, modular, económico y de fácil implantación; que abarca tres aspectos esenciales: la ecológica (utilizar la productividad de la naturaleza sin dañarla); la económica (aseguramiento de los recursos necesarios para el consumo humano); y la social (fomentar el beneficio social a través del confort). 

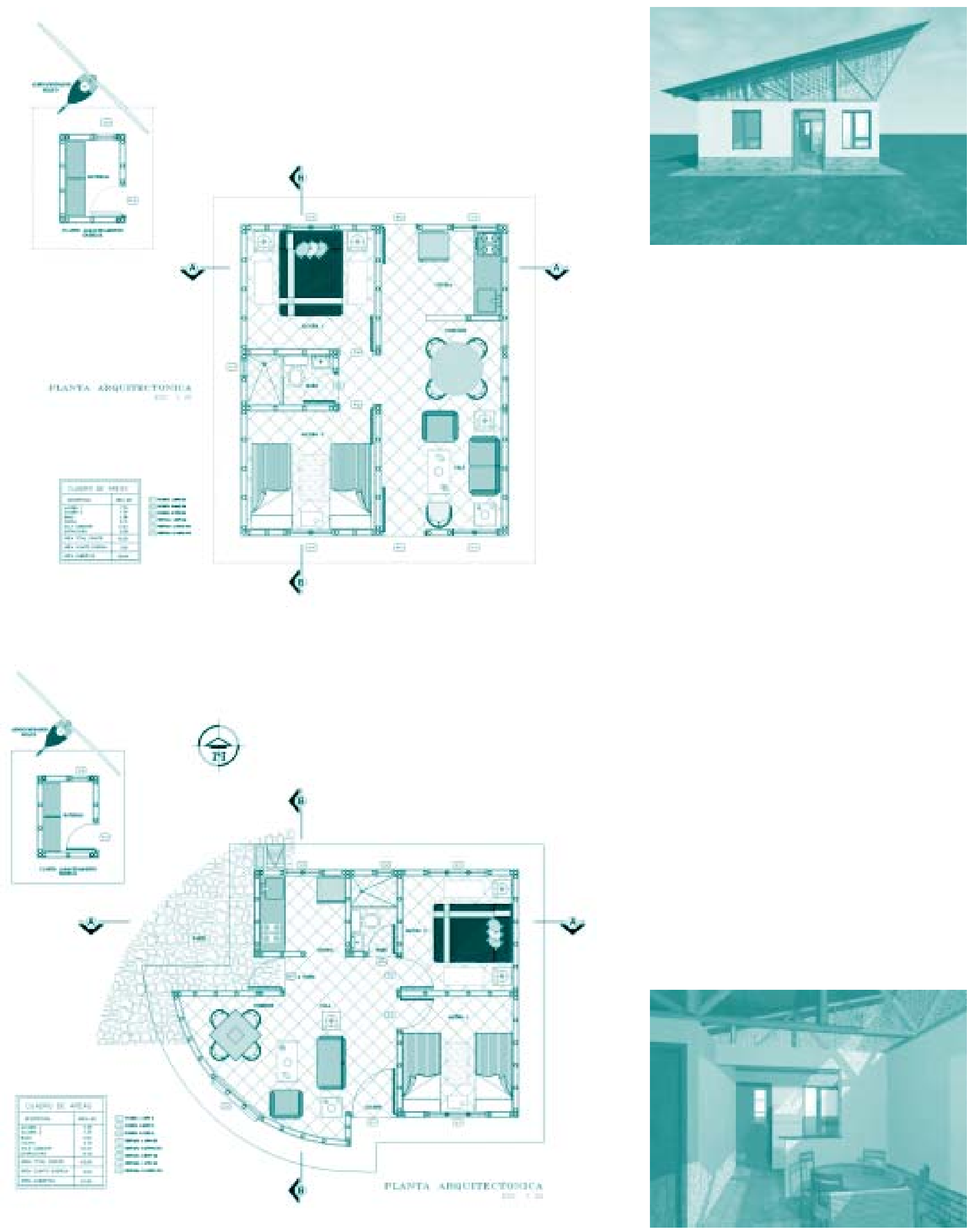
- El proyecto incorpora en forma integral conceptos como: respeto al entorno, comunidades verdes, organización y participación comunal, comunidad como módulo de diseño, control social de los servicios, uso racional de la energía, ahorro de agua, manejo integral de desechos líquidos y sólidos, industrialización y modulación en sitio, confort, seguridad, sanidad y calidad de uso. En suma, reducción sustancial en los costos y mejoramiento en la calidad de vida, mediante un uso racional y ambiental de los recursos incorporados.

- La vivienda será construida en guadua, en el sistema de bahareque embutido, es decir, esterilla y recubrimiento con arcilla estabilizada con cal pura, que permite una cámara de ventilación en los muros, un aislamiento térmico y acústico, y crea un confort al interior de la vivienda, así mismo las ventanas y especial una abertura superior en la cubierta, permiten crear efectos venturi al interior de la misma, es decir, recirculación de la ventilación natural. Su cubierta en teja de barro, permite la recolección de aguas Iluvias las cuales son almacenadas en un tanque subterráneo, a él llegan las aguas del nacimiento o del punto de agua dado por el municipio. Ver plano arquitectónica para vivienda rural (1) y vivienda suburbana (2).

- La vivienda viene acompañada de un proceso de capacitación en la utilización del sistema constructivo de bahareque embutido o encementado donde el campesino aporta con su mano de obra no calificada una ayuda en la construcción de su propia vivienda, para crear un sentido de apropiación y respeto ambiental.

- La vivienda actúa como elemento articulador en generación de empleo por los cultivos orgánicos, en el control de enfermedades por sus sistemas de ventilación e iluminación y en la aplicación de nuevas tecnologías para el caso del aerogenerador eólico y de la planta de tratamiento de aguas residuales PTAR. Ver dibujo anexo.

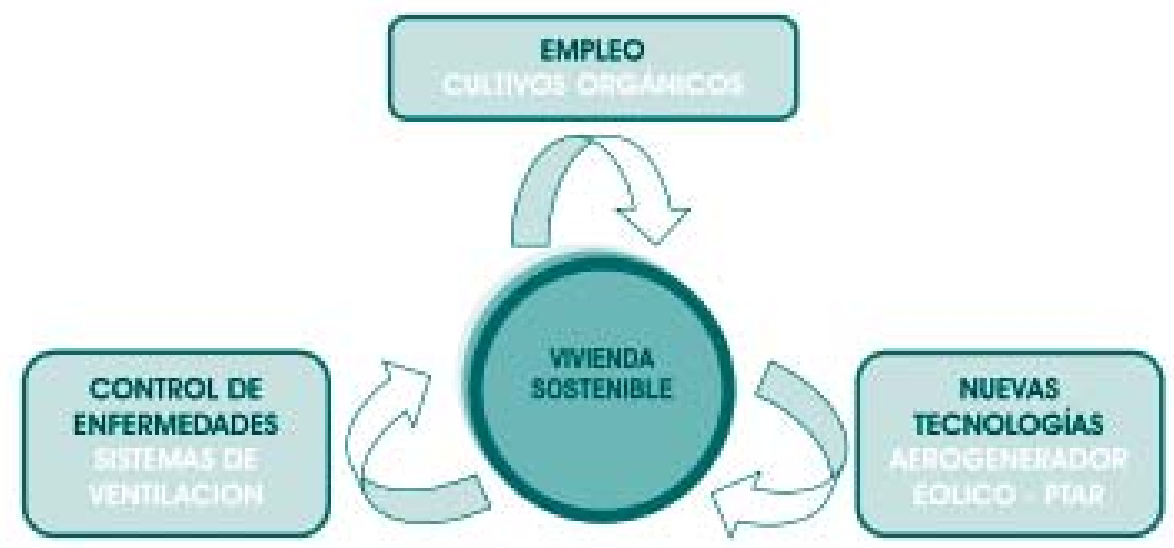

\section{CAPACITACIÓN EN CULTIVOS ORGÁNICOS}

- Entiéndase como agricultura orgánica o ecológica, las prácticas de cultivo que propenden por una producción agraria sostenible en relación con el medio ambiente, recursos naturales, biodiversidad y el mismo hombre. Su fin último es la no utilización de insumos externos como plaguicidas y fertilizantes de síntesis química o transgénicos, a cambio de realizar las prácticas fitosanitarias y de producción a partir de procesos y controles naturales o biológicos, en busca de obtener producciones limpias, de mayor calidad nutricional, inocuas, que no generen problemas a la salud humana, y preserven el entorno o ecosistema. 
- Esta corriente de agricultura sostenible, busca que los alimentos vuelvan a tener los sabores y olores propios de la naturaleza, que den placer y agrado tanto a humanos como animales. En Colombia, dado el interés y crecimiento del consumo y producción de productos ecológicos, el Ministerio de Agricultura y Desarrollo Rural, expidió la Resolución No 0074 de 04 Abril de 2002, por medio de la cual se establece el reglamento para la producción primaria, procesamiento, empacado, etiquetado, almacenamiento, certificación, importación y comercialización de productos agropecuarios ecológicos.

- Se propone iniciar con cultivos de stevia, que es un endulzante natural de fácil siembra y recolección, en lugares destinados a la siembra de hoja de coca pues su retiro es igual al utilizado por los campesinos "raspachines".

- Así mismo, realizar cultivos de cacao, por su fácil adaptación y producción continua durante todo el año; y por sus valores medicinales y de sabor que en estos momentos satisfacen mercados internacionales.

- Establecer una organización de la comunidad en una cooperativa de trabajo asociado, cuya característica principal es la capacitación en los cultivos de stevia y cacao, con una única finalidad de producción sostenible y exportación de calidad.

\section{MANEJ O DE RESIDUOS SÓLIDOS Y LÍQUIDOS}

- La vivienda cuenta con un sistema de reciclaje del agua jabonosa, en el tanque sanitario, para ello pasa por un sistema de filtros antes de llegar a la trampa de grasas, el cual cuenta con un nivel de rebose, que desvía las aguas por exceso ya tratadas a un tanque de homogenización o tanque primario, cuya finalidad es recoger todas las aguas residuales y sólidos de la vivienda.

- Cabe anotar que la tubería de aguas jabonosas y de aguas negras no se encuentra hasta el tanque de homogenización. En la caja de trampa de grasas se encuentra una bomba sumergible que proporciona la recirculación del agua, hacia la vivienda. El tanque de homogenización posee una bomba sumergible que conduce el agua hacia las cámaras de aireación, y de allí por manejo de niveles al tanque sedimentador y finalmente al tanque de sulfato de plata, de donde sale un agua tratada apta para cultivos orgánicos; el sedimentador cuenta con una caja para el secado de lodos, procedentes de la materia fecal humana y de material orgánico producto de los alimentos.

- Del tanque subterráneo sale una tubería que irriga los cultivos orgánicos, por el sistema de gota a gota, que permite optimizar y racionar el agua en estos sistemas de cultivos, en este sistema prima la gravedad y el agua se desliza por entre los cultivos. Ver plano detalle Ptar (3)

- La vivienda cuenta con un tanque de almacenamiento de agua potable que recibe los puntos de agua dados por el municipio, o como producto de recolección de aguas lluvias procedentes de la cubierta, o por aljibes cercanos a la vivienda, su suministro al tanque de $250 \mathrm{I}$. se realiza por medio de una bomba sumergible.

- El proyecto cuenta con una capacitación en el manejo y tratamiento de las máquinas utilizadas, así como del tratamiento de purificación del agua residual, para su posterior utilización en los cultivos orgánicos. 


\section{AEROGENERADOR EÓLICO}

- El suministro de energía eléctrica para la vivienda y las bombas sumergibles del tanque de homogenización, la trampa de grasas y el tanque de almacenamiento de agua potable; serán suministrados por una turbina eólica, con una capacidad de suministro para tres viviendas.

- Se debe realizar un estudio de micro zonificación eólica, para establecer las condiciones del viento del lugar, con el fin de conocer las condiciones y la altura a las cuales deben funcionar las máquinas.

- La energía es almacenada en el cuarto de baterías para su uso en los requerimientos anteriormente descritos.

- Se obtiene una energía limpia no contaminante con el medio ambiente y sin afectación e impactos negativos al contexto rural o suburbano.

- Se capacita al campesino para reportar inconvenientes a la cooperativa de trabajo asociado, en el funcionamiento normal de la turbina eólica o del deterioro de las baterías por su uso continuo.

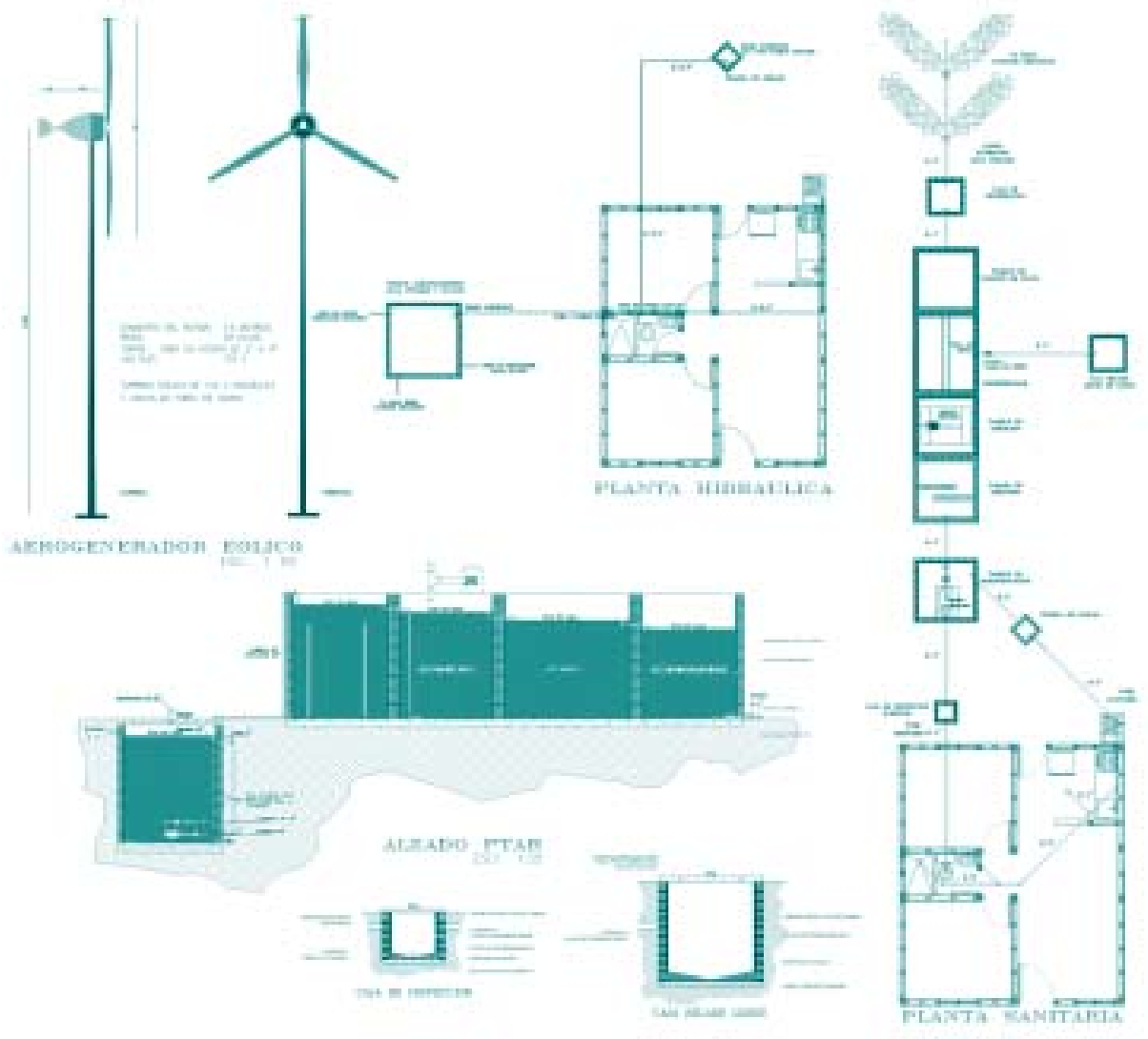

\section{RESULTADOS ESPERADOS}

- Concebir un prototipo de vivienda rural autosuficiente como un ente biológico.

- Los gastos económicos por los servicios básicos, se transformaran en ahorro para el campesino. 
- Formar, en el campesino, la actitud del manejo y aplicación de nuevas tecnologías.

- Acompañar el proyecto de un proceso de capacitación, para el adecuado manejo de cultivos.

- Mejorar la calidad de vida del hombre del campo.

- Participación de la comunidad, en la ejecución de su proyecto habitacional.

- La aplicación de conceptos de integración de comunidad, hábitat ambiental y desarrollo socioeconómico focales.

- Búsqueda de una economía local sostenible, que produzca un hábitat protegido, generador de empleo y riqueza, para un desarrollo rural.

- Destinación del gasto público para gestión de agua, agricultura, infraestructura e equipamientos, rurales.

- Análisis socioeconómico del espacio y de los usos del territorio, con una visión tanto de presente como de futuro.

- Los procesos de seguimiento continuado y de evaluación -en distintos momentos, inicial, intermedia y final- deben abordar, además aspectos ambientales y sociales, en aras de valorar la contribución del instrumento al desarrollo sostenible del espacio.

- Realizar jornadas de educación ambiental, estudios de intervención en espacios naturales, jornadas de sensibilización para la población y los visitantes.

- Integración real, por capacitación, intervención rural en construcción y seguimiento periódico con miras a mejorar las técnicas aplicadas.

\section{PROPUESTA}

\section{CONSTRUCCIÓN DE VIVIENDA NUEVA}

- Construcción de una vivienda sostenible en bahareque embutido o encementado, con la aplicación de tecnologías limpias, en una Ptar residencial y un aerogenerador eólico.

- Implementación de cultivos orgánicos y su capacitación en su producción, para lo cual se organiza la comunidad en cooperativa.

- Creación integral de un ecosistema participativo hombre-medio ambiente, mediante procesos de capacitación acompañados por folletos para la vivienda sostenible, y aplicación de tecnologías limpias.

- Reducción de enfermedades y por consiguiente destino de estos rubros a capacitación constante en nuevas tecnologías y tecnificación de la mano de obra en actividades no convencionales 